\title{
Stratégie de la FMH pour 2021-2024
}

\author{
Nora Wille ${ }^{a}$, Ursina Pally Hofmann ${ }^{b}$, Yvonne Gillic
}

a Dre phil., collaboratrice scientifique de la présidente; ${ }^{b}$ Dre iur., secrétaire générale de la FMH; ${ }^{c}$ Dre méd., présidente de la FMH

Le 27 mai 2021, date à laquelle la Chambre médicale a approuvé la stratégie de la FMH pour la législature 2021-2024, a marqué la fin d'un processus de genèse entamé en 2019. En même temps, la décision de la Chambre médicale marque le début de la phase décisive du processus stratégique, à savoir la mise en œuvre. Dans l'article qui suit, nous passons en revue la genèse et les contenus de la stratégie appelée à constituer le fil rouge de notre activité dans les années à venir.

Au début de chaque nouvelle législature, lorsqu'il définit les objectifs politiques et stratégiques pour les années qui viennent, le Comité central de la FMH se repose les mêmes questions clés, déterminantes pour l'activité de la FMH: quels sont les défis essentiels se posant à l'association? Quels sont nos objectifs? Comment cibler et organiser notre travail pour nous permettre d'atteindre ces objectifs, ou tout au moins nous en approcher? Très rapidement, on se rend compte dans le cadre de ce processus que la définition de la stratégie est bien plus qu'un simple exercice statutaire pour le Comité central [1], et qu'elle remplit une diversité de fonctions. Elle offre un espace propice à une réflexion approfondie et met l'accent sur les priorités communes; elle communique clairement à l'égard du public les principaux objectifs du travail de l'association et définit un fil rouge pour la législature qui s'ouvre, destiné à guider le travail et à évaluer le degré de réalisation des objectifs.

Pour pouvoir remplir cette fonction, le processus stratégique suit un déroulement clair (cf. fig. 1). Après une analyse approfondie des défis qui l'attendent, la FMH en déduit les objectifs qui devront guider son travail, puis les mesures grâce auxquelles elle entend atteindre les

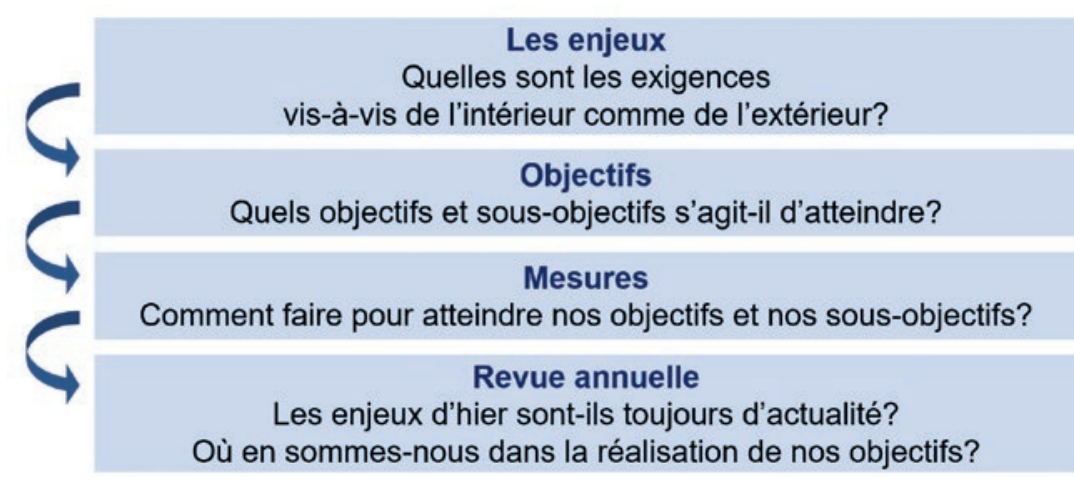

Figure 1: Le processus stratégique de la FMH. objectifs et sous-objectifs qu'elle se sera fixés. Durant la législature, le Comité central vérifie une fois l'an si les défis et les objectifs sont toujours d'actualité, et examine l'état de mise en œuvre des mesures.

\section{Point de départ: \\ Quelles sont les attentes de nos membres et des autres parties prenantes?}

Pour que la stratégie réponde le mieux possible aux défis clés se posant au système de santé et aux attentes de ses membres, la FMH a commencé par recenser de manière systématique les propositions des diverses parties prenantes. 3000 membres tirés au sort, les déléguées et délégués à la Chambre médicale, les cadres du Secrétariat général de la FMH et plusieurs expertes et experts ont été interrogés afin de définir un ordre de priorité entre les principaux défis qui attendent la FMH. Le sondage a été effectué durant le dernier trimestre 2019. Ses résultats étaient attendus avant les élections initialement prévues pour mai 2020, mais la crise du coronavirus est arrivée et avec elle le report des élections.

Les résultats du sondage [2], publiés en septembre 2020, ont montré que tant les médecins que les experts considèrent que c'est l'une des tâches centrales de la FMH que de faire valoir les points de vue du corps médical en politique. Et l'une des préoccupations essentielles de ce dernier est le maintien de l'autonomie tarifaire, actuellement remise en question par les plans de budgétisation des coûts de la santé. Il faut tout entreprendre pour prévenir les conséquences néfastes prévisibles du "pilotage des coûts» et des "objectifs de coûts" pour la prise en charge médicale et donc pour le travail quotidien des médecins. Mais la prise en charge médicale fait face à de nombreux autres défis: les thèmes retenus par les personnes ayant participé au 
(1) Politique suisse de la santé : représentation des intérêts

(2) Tarifs ambulatoires et maintien de l'autonomie tarifaire

(3) Conditions de travail et attrait de la profession

(4) Cohésion / présentation d'un front uni

(5) Volume des tâches administratives

(6) Pression temporelle sur la relation médecin-patient

(7) Pression sur les coûts et prise en charge médicale

(8) Pénurie de médecins et garantie de la prise en charge

(9) Formation prégraduée, postgraduée et continue

(10) Image du corps médical

(11) Conciliation vie familiale/professionnelle

(12) Admission/liberté de contracter/obligation de contracter

(13) Représentation des positions de la FMH dans les médias

(14) Santé des médecins et burnout

Figure 2: Enjeux cardinaux de la FMH durant la législature 2021-2024.

sondage sont donc non seulement les conditions de travail et l'attrait de la profession, mais également des aspects plus concrets comme la pression temporelle dans la relation médecin-patient, la pression sur les coûts, l'augmentation des tâches administratives, sans oublier la conciliation vie familiale/vie professionnelle, la santé des médecins et le burnout.

Références

1 Conformément à l'article 49, alinéa 2 , des Statuts de la FMH, l'une des tâches du Comité central, en tant qu'organe directeur de la FMH, est la "définition des objectifs politiques et stratégiques».

2 Wille N, Schlup J. Quels sont les principaux défis qui atten dent la FMH? Bull Med Suisses. 2020; 101(40):1254-8; URL: https://bullmed.ch/ article/doi/ saez.2020.19251

\section{Des résultats du sondage à la stratégie: définition des défis prioritaires}

Le nouveau Comité central, élu le 28 octobre 2020, a commencé par consolider les résultats du sondage en une liste de défis prioritaires (cf. fig. 2). Lors d'un atelier organisé en décembre 2020, ses membres ont discuté et classé par ordre de priorité les propositions remises par les membres de la FMH et les délégué-e-s à la Chambre médicale, complétées par les avis des expert-e-s interrogés. Ils se sont concentrés sur les thèmes présentant un intérêt non pas pour certains sous-groupes, mais pour tous les membres, ainsi que ceux qui ne peuvent pas raisonnablement être traités par l'une ou l'autre or-

\section{Politique et communication, Dre Yvonne Gilli}

La FMH œuvre pour un corps médical uni et s'engage pour des soins médicaux forts.

Données, démographie et qualité, Dr Christoph Bosshard

La FMH s'investit pour la transparence dans les activités qualité et pour le maintien de l'autonomie en matière de qualité en s'appuyant sur des données fiables.

\section{Prestations et développement professionnel, Dr Michel Matter}

La FMH façonne activement l'avenir de la profession médicale et fournit des prestations attractives à ses membres.

Médecine et tarifs ambulatoires, Dr Urs Stoffel

La FMH s'investit pour préserver l'autonomie tarifaire, pour assurer une rémunération adéquate et pour renforcer la prise en charge ambulatoire.

Médecine et tarifs hospitaliers, Dre Jana Siroka

La FMH s'engage pour une rémunération adéquate et pour de bonnes conditions-cadres régissant l'activité médicale dans le secteur hospitalier.

\section{Numérisation / eHealth, Dr Alexander Zimmer}

La FMH s'investit pour une numérisation adaptée aux besoins de la prise en charge médicale et véritablement utile.

Santé publique et professions de la santé, Dr Carlos Quinto

La FMH soutient et renforce la santé publique dans le sens souhaité par les médecins.

Secrétariat général, Dre iur. Ursina Pally Hofmann

Le SG administre, coordonne et développe la FMH en tant qu'organisation experte.

Figure 3: Objectifs principaux des départements et du Secrétariat général

pour la législature 2021-2024.

ganisation membre, mais exigent des efforts concertés à l'échelle du pays. Le Comité central a par ailleurs aussi examiné les préoccupations évoquées par les membres à la lumière des derniers développements et des attentes de groupements d'intérêts externes. Pour pouvoir offrir une plus-value en tant qu'association professionnelle, la FMH ne peut pas se contenter de recenser les besoins de ses membres, mais doit utiliser son expertise pour anticiper les développements, s'y préparer et s'attaquer aux problèmes avant qu'ils n'aient des répercussions pour les membres.

\section{Grâce aux objectifs, les défis deviennent des mandats aux départements de la FMH}

Pour traduire ces défis en tâches concrètes, confiées aux organes de la FMH, il est nécessaire de définir des objectifs. C'est la raison pour laquelle l'étape suivante a consisté pour les départements à évaluer quels objectifs stratégiques devaient être les leurs au vu des défis, classés par ordre de priorité, qui se poseront à l'organisation dans les années qui viennent. Les propositions élaborées par les responsables des départements et par la secrétaire générale ont été présentées, discutées et adoptées (cf. fig. 3) lors d'un deuxième atelier, courant janvier 2021. Alors que les objectifs principaux définissent des axes d'ordre général, les quatre à cinq sous-objectifs correspondant à chacun d'eux sont de nature nettement plus concrète (ils ne sont pas reproduits dans la figure 3 pour des raisons de place).

La dernière étape de la mise en œuvre opérationnelle a été bouclée dans le cadre du troisième atelier stratégique, en février 2021, au cours duquel le Comité central et l'ensemble des cadres de la FMH ont attribué une ou plusieurs mesures à chaque sous-objectif. Cette concrétisation opérationnelle de la manière dont la FMH compte relever les défis qui se posent à elle constituera le fil rouge de la présente législature. Elle constitue également la base de l'évaluation annuelle examinant les progrès accomplis pour chacune des mesures retenues.

Les objectifs stratégiques de la FMH ont été présentés aussi bien lors de l'Assemblée des délégués que de la Chambre médicale. Les membres de l'Assemblée des délégués, qui s'est tenue le 24 mars 2021, ont salué la mise au point de la stratégie et - après une discussion durant laquelle ils ont donné des suggestions de mise en œuvre - ont recommandé à la Chambre médicale de l'adopter, en vertu de l'article 30, alinéa 2, des Statuts. Les membres de la Chambre médicale, réunis le 27 mai 2021, ont approuvé la stratégie à 146 voix, avec 3 oppositions et 5 abstentions, donnant par là même le coup d'envoi de la phase de mise en œuvre. 